\title{
ULTRACYTOCHEMICAL LOCALIZATIONS OF ADENOSINE NUCLEOTIDASE ACTIVITIES IN THE HUMAN TERM PLACENTA, WITH SPECIAL REFERENCE TO 5'-NUCLEOTIDASE ACTIVITY
}

\author{
Shigeki MATSUBARA *, TARo TAMADA*, KoIchi KURAHASHI ***. \\ and Takuma SAITO **
Departments of Obstetrics and Gynecology * and Anatomy **, Jichi Medical School, Minamikawachi-machi, Tochigi 329-04 and Department of Biology, Osaka Dental University ${ }^{* * *}$, Kyobashi, Osaka 573

Received for publication April 20, 1987 and in revised form June 26, 1987

The ultracytochemical localizations of adenosine nucleotidase activities were investigated in the human term placenta. The enzymes studied were as follows: $5^{\prime}$-nucleotidase $\left(5^{\prime}-\mathrm{N}\right), \mathrm{Ca}^{++}$-activated adenosine triphosphatase $\left(\mathrm{Ca}^{++}\right.$ATPase), $\mathrm{Mg}^{++}$-activated adenosine triphosphatase $\left(\mathrm{Mg}^{++}\right.$ATPase $)$and nucleotide diphosphatase (NDPase). $\quad 5^{\prime}-\mathrm{N}$ activity was demonstrated using not only the lead nitrate method of Wachstein and Meisel but also the recently developed cerium and neodymium method as well.

The reaction products for $5^{\prime}-\mathrm{N}$ activity were found on the external surface of the microvillous plasma membrane of the syncytiotrophoblast. Alpha-betamethylene adenosine diphosphate (AOPCP), at a concentration of $2.0 \mathrm{mM}$, effectively inhibited $5^{\prime}-\mathrm{N}$ activity. $\mathrm{Ca}^{++} \mathrm{ATPase}$ and $\mathrm{Mg}^{++} \mathrm{ATPase}$ activity were observed strongly on the microvillous membrane of the syncytiotrophoblast and weakly on the basal plasma membrane of the syncytiotrophoblast. NDPase, using ADP as a substrate, was localized on the microvillous membrane.

These observations suggest that the syncytiotrophoblast is active in the nucleotide metabolism and that microvillous surface of the syncytium may play an important role in regulating the feto-placental-maternal microcirculation in the human term placenta.

Both biochemical and cytochemical properties of the $\mathrm{Ca}^{++}$and/or $\mathrm{Mg}^{++}$ stimulated adenosine nucleotidases (ATPase, NDPase and $5^{\prime}-\mathrm{N}$ ) have already been demonstrated in the vascular endothelium and in the vascular smooth muscle $(8,18$, 19). These nucleotidases sequentially degrade ATP to ADP, AMP and finally to adenosine. Speculation has been made as to the presence of these nucleotidases in the placenta on biochemical grounds (2, 9, 13, 21, 24), and these enzymes may play the following three important roles in placental physiology.

First, the placental nucleotidase system may modulate platelet aggregation, and thus control placental microcirculation. It is well accepted that adenosine inhibits platelet aggregation, while ADP activates it contrarily. The mechanism which 
regulates platelet aggregation in the placenta is still obscure, but it has been demonstrated that placental NDPase and $5^{\prime}-\mathrm{N}$ may play an antiaggregatory role by degrading ADP to AMP, finally to adenosine $(2,9)$.

Second, this nucleotidase system, especially $5^{\prime}-\mathrm{N}$, may modulate feto-placentalmaternal vasoactivity. Reports have so far accumulated suggesting the importance of adenosine as a potent vasodilator in the heart tissue (3). The factors that mediate the local regulation of the blood flow in the human placenta, a non-innervated organ, have not been fully elucidated, but vasoactive adenosine in the placenta could be of physiological significance in maintaining feto-placental-maternal microcirculation $(10,22)$ similar to adenosine in the heart (3).

Third, it has been reported that adenosine, generated by the nucleotidase system, may alter adenylate cyclase (ACLase) activity (12), which is shown to be an important regulatory system of the human placental function (16).

In these contexts, it would be worth investigating the nucleotidase activities in the human placenta. Some biochemical data are available about the properties of these enzymes in the placenta $(2,9,13,21,24)$, but, to the best of our knowledge, direct cytochemical evidence of the localizations of these enzymes has not been reported as yet. In the present study, the ultracytochemical localizations of adenosine nucleotidases $\left(\mathrm{Ca}^{++}\right.$ATPase, $\mathrm{Mg}^{++}$ATPase, NDPase and 5'-N) were investigated, with special reference to that of $5^{\prime}-\mathrm{N}$ activity. At the same time, alpha-betamethylene adenosine diphosphate (AOPCP) was applied cytochemically as a potent inhibitor of $5^{\prime}-\mathrm{N}$ activity. This is the first report of the application of AOPCP to cytochemistry.

\section{MATERIALS AND METHODS}

Human term placentas from healthy women who maintained an uncomplicated obstetrical course were obtained immediately after delivery. Several pieces of the tissue were fixed in cold 1.5-2.0\% glutaraldehyde in a $0.1 \mathrm{M}$ cacodylate buffer, $\mathrm{pH} \mathrm{7.2,} \mathrm{for} 30 \mathrm{~min}$. After overnight washing in a cacodylate buffer, $40 \mu \mathrm{m}$ sections were made with the use of a Vibratome or a freezing microtome and then incubated in the following reaction media for $30 \mathrm{~min}$ at $37^{\circ} \mathrm{C}$. For $5^{\prime}-\mathrm{N}$ activity, the lead nitrate method (Wachstein and Meisel [23]), the cerium method (Robinson and Karnovsky [20]) and the neodymium method (Kurahashi ad Maeda [11]) were used. In the

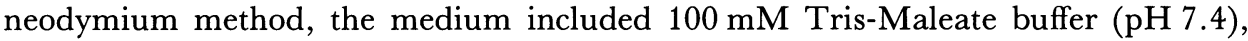
$1 \mathrm{mM}$ 5'-AMP, $2 \mathrm{mM} \mathrm{MgCl}_{2}, 2 \mathrm{mM}$ neodymium chloride. For the cytochemical detection of other nucleotidase activities, the methods used were as follows: the lead citrate method (Ando et al. [1]) for $\mathrm{Ca}^{++}$ATPase, lead nitrate method (Wachstein and Meisel [23]) for $\mathrm{Mg}^{++}$ATPase and lead nitrate method (Novikoff and Goldfischer [17]) for NDPase. As for NDPase activity, ADP was used as a substrate. In almost every procedure, 1-p-bromotetramisole oxalate (Sigma) was added as an alkaline phosphatase inhibitor $(4,14)$ into the reaction media adjusted to $1.0 \mathrm{mM}$ in the final concentration. To further rule out the contamination of alkaline phosphatase activity the sections were preincubated in a cacodylate buffer containing $1.0 \mathrm{mM}$ bromotetramisole and then incubated in the reaction media (14). After the incubation, for electron microscopy, the sections were postfixed in a cold Caulfield's solution (1\% osmium tetroxide) for $60 \mathrm{~min}$, dehydrated in a series of graded alcohols and 
embedded in epoxy resin, Quetol 812 (Nissin EM Co. Tokyo, Japan). Ultrathin sections were prepared with an LKB ultrotome, unstained or stained with uranyl acetate and lead citrate, and observed under a Hitachi $\mathrm{H}-700$ or an HU-12A electron microscope.

As a cytochemical control for each enzyme activity, the reaction medium was altered as follows: 1) substrate (ATP, ADP or AMP) was omitted, 2) betaglycerophosphate was used as a substrate. To confirm the cytochemical specificity further, the following were also performed. For $5^{\prime}-\mathrm{N}$ activity, 3) AOPCP, which is an $\mathrm{ADP}$ analogue and a potent inhibitor of $5^{\prime}-\mathrm{N}$ activity (6), was added to the final concentration of $0.2-2.0 \mathrm{mM}$, and 4) AOPCP was substituted for AMP as a substrate. As for $\mathrm{Ca}^{++}$ATPase activity, 3) $\mathrm{Ca}^{++}$was eliminated, and 4) $10 \mathrm{mM} \mathrm{Ca}^{++}$was replaced by 0.1 or $1.0 \mathrm{mM} \mathrm{Ca}^{++}$.

\section{RESULTS}

1. Ultracytochemical Localization of $5^{\prime}-\mathrm{N}$ and the Effect of AOPCP to 5'-N Activity

The reaction products for $5^{\prime}-\mathrm{N}$ activity were demonstrated on the plasma membrane of the microvilli of the syncytiotrophoblast (Figs. 1-3). Precipitates could also be observed on the membrane of the pinocytotic vesicles of the syncytiotrophoblast. Activity was negative on the basal plasma membrane of the syncytiotrophoblast, on the plasma membrane of the cytotrophoblast and on the villous stroma. The lead nitrate method, the cerium method and the neodymium method gave exactly the same results as far as the localization of $5^{\prime}-\mathrm{N}$ activity was concerned. However, with the use of the lead nitrate method, the reaction products were often not uniformly distributed on the plasma membrane. They were observed rather discontinuously (Fig. 3). On the other hand, with the cerium and the neodymium method, 5'-N activity was shown more continuously on the plasma membrane of the microvilli (Figs. 1, 2).

Partial reduction in the activity was brought on by the addition of $0.5 \mathrm{mM}$ AOPCP and complete inhibition with $2.0 \mathrm{mM}$ AOPCP (Figs. 4a-c). Substituting AOPCP for AMP, the reaction was negative (Fig. 5a), so AOPCP might not be hydrolyzed by $5^{\prime}-\mathrm{N}$. Controls incubated in a substrate free medium (Fig. 5b) or in a medium substituting beta-glycerophosphate for AMP (Fig. 5c) gave no reaction.

2. Ultracytochemical Localizations of $\mathrm{Ca}^{++} \mathrm{ATPase}, \mathrm{Mg}^{++} \mathrm{ATPase}$ and NDPase

The reaction products for $\mathrm{Ca}^{++} \mathrm{ATPase}$ activity were observed strongly on the microvillous membrane of the syncytiotrophoblast. Weak activity could also be found on the basal plasma membrane and on the plasmalemma of the pinocytotic vesicles of the syncytiotrophoblast (Figs. 6a, b). Fine precipitates could also be observed on the cytotrophoblastic plasma membrane, but these precipitates were discontinuously distributed on the membrane, and they were very faint (Fig. 6c.). Therefore, further study is required to elucidate whether these precipitates are true enzymatic reactions or not. At $0,0.1$ or $1.0 \mathrm{mM} \mathrm{Ca}^{++}$, no activity was found. In the substrate free control and in the beta-glycerosphate control, the precipitates were not recognized. $\mathrm{Mg}^{++}$ATPase activity was localized mainly on the microvillous membrane of the syncytium (Fig. 7). As for NDPase, the activity was confined to the apical microvillous membrane of the syncytiotrophoblast (Fig. 8). The activity disappeared in the substrate free control or in the beta-glycerophosphate control. In the present ex- 


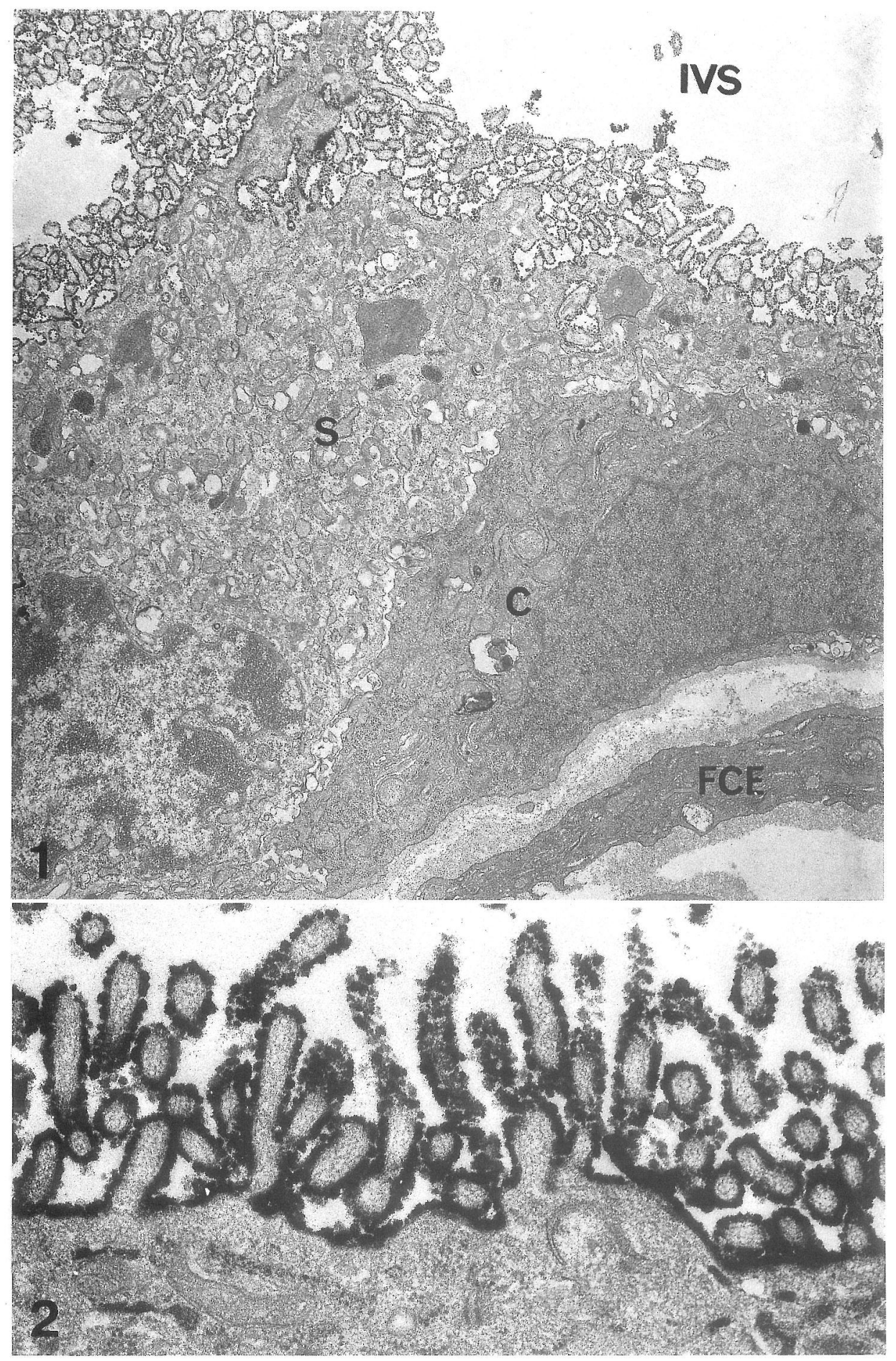







perimental condition, $\mathrm{Ca}^{++}$ATPase, $\mathrm{Mg}^{++}$ATPase and NDPase activity were negative in the cytoplasm of the syncytio- and cyto-trophoblast, nor on the villous stroma.

\section{DISCUSSION}

The purpose of this paper is to describe the ultracytochemical localizations of adenosine nucleotidase activities, especially that of $5^{\prime}-\mathrm{N}$ activity.

AOPCP was discovered by Burger and Lowenstein as a potent inhibitor of intestinal smooth muscle $5^{\prime}-\mathrm{N}$ activity (6). Some other researchers biochemically demonstrated that human placental $5^{\prime}-\mathrm{N}$ was also inhibited by AOPCP (13). However, to the best of our knowledge, the cytochemical application of this substrate has not been reported as yet. The present study showed that AOPCP could be applied cytochemically as a potent inhibitor of $5^{\prime}-\mathrm{N}$ activity at an ultrastructural level.

The presence of $5^{\prime}-\mathrm{N}(13), \mathrm{Ca}^{++}$ATPase (24), $\mathrm{Mg}^{++}$ATPase (21) and NDPase $(2,9)$ in the placenta has already been shown, but direct cytochemical evidence of their localizations in this tissue has been lacking. The present study demonstrated that $5^{\prime}-\mathrm{N}$ activity was exclusively confined to the microvillous membrane of the syncytiotrophoblast. Concerning this localization, the lead nitrate, the cerium and the neodymium method yielded the same results. All the cytochemical control studies confirmed the specificity of the enzyme activity. Further, the other nucleotidases were also localized mainly on the microvillous membrane of the syncytium. In other words, all the nucleotidases studied here coexisted on the microvilli adjacent to the maternal circulation. This fact tempted us to derive the following three assumptions.

First, the placental nucleotidase system may modulate the platelet aggregation in the intervillous space of the human placenta. Recently, Ogawa et al. demonstrated the ultrastructural localizations of $\mathrm{Ca}^{++} \mathrm{ATPase}$ and $\mathrm{Na}^{+}, \mathrm{K}^{+}$-ATPase in the rat aortic endothelium, and they suggested that the nucleotidase system might play an important role in regulating platelet aggregatory activity in the aortic endothelium (18).

The mechanism that regulates platelet aggregation in the placenta has not been elucidated, but, recently, Barradas et al. demonstrated that the human placenta had a potent antiaggregatory activity, and that this activity was due to ADP degradation to

FIG. 1. Ultracytochemical localization of $5^{\prime}-\mathrm{N}$ in the human term placenta. The cerium method. $\times 11,000$

Activity was localized on the plasma membrane of the microvilli of the syncytiotrophoblast (S). Precipitates could also be observed on the plasmalemma of the pinocytotic vesicles of the syncytium. IVS: Intervillous Space, C: Cytotrophoblast, FCE: Fetal Capillary Endothelium.

FIG. 2. 5'-N activity. The neodymium method. $\times 38,000$ Intense activity was confined to the external surface of the microvillous membrane.

FIG. 3. Ultracytochemical localization of $5^{\prime}-\mathrm{N}$ activity in the human term placenta. The lead nitrate method. $\quad \times 25,000$

Reaction products were observed on the microvillous membrane. The rection products were distributed rather discontinuously.

Figs. 4a-c. Inhibition of $5^{\prime}-\mathrm{N}$ activity by AOPCP. The cerium method. $\times 27,000$

a. At $0.2 \mathrm{mM}$ AOPCP. Intense $5^{\prime}-\mathrm{N}$ activity was still positive on the plasma membrane.

b. At $0.5 \mathrm{mM}$ AOPCP. Activity was partly reduced.

c. At $2.0 \mathrm{mM}$ AOPCP. Activity was completely inhibited. 

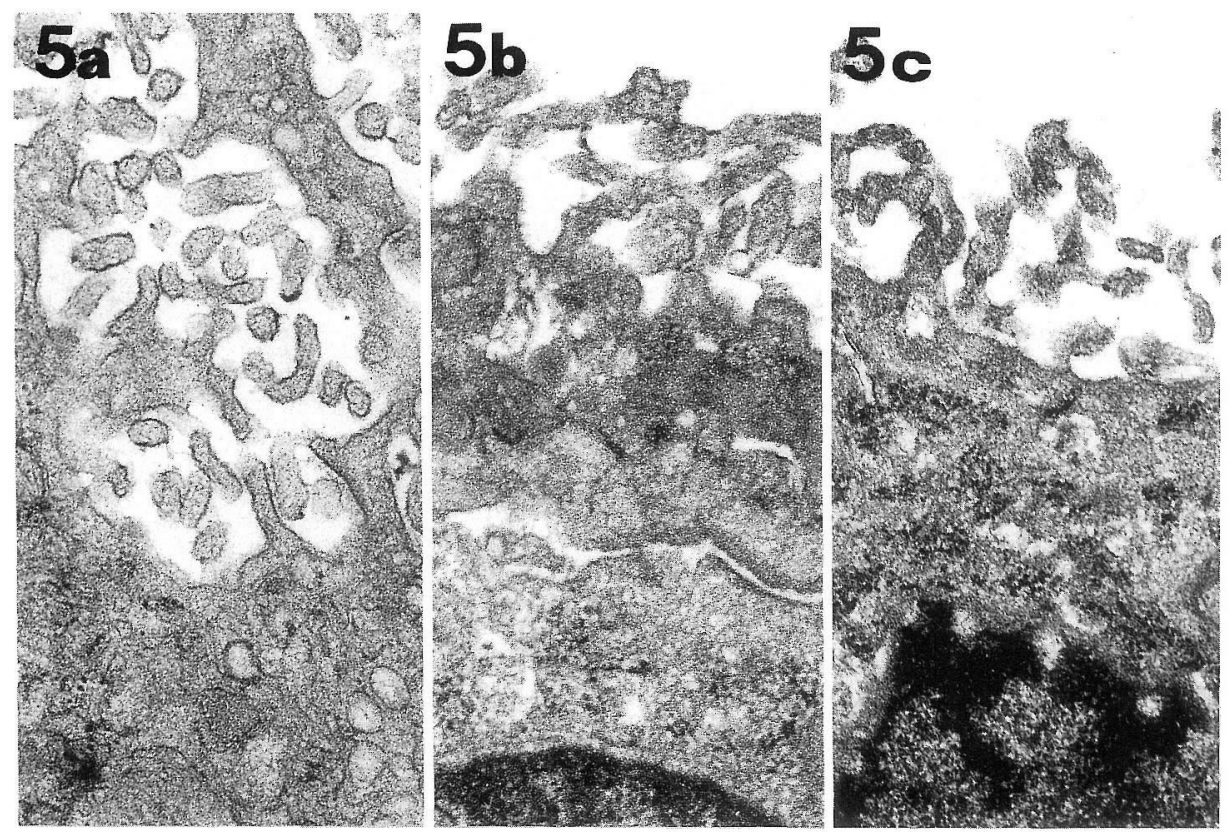

FIGS. 5a-c. Control experiments for 5'-N activity. The cerium method. $\times 27,000$

a. The reaction was completely abolished when AOPCP was substituted for AMP.

b. No reaction was found in a substrate free control.

c. The reaction products were not found with beta-glycerophosphate as a substrate.

adenosine by NDPase and 5 - $\mathrm{N}$ (2). This experimental observation, combined with the assumption by Ogawa et al. (18), leads us to believe that placental nucleotidase system may also regulate the platelet aggregatory activity similar to that in the rat aortic endothelium.

Second, this nucleotidase system, especially $5^{\prime}-\mathrm{N}$, may modulate the feto-placental-maternal vasoactivity. The presence of adenosine in the human placenta was reported by Sim and Maguire (22) and some others also suggested that adenosine might play a role in maintaining placental microcirculation (10). In the heart, it is well known that adenosine, a potent vasodilatory agent, acts as a mediator of metabolic regulation of the coronary circulation and functions as an agent that can rapidly adjust coronary flow to changing oxygen requirements of the heart (3). In this regulation, $5^{\prime}-\mathrm{N}$ is believed to play the role of an adenosine producing system (3).

Placental blood flow is essential to fetal growth and fetal well-being, and it is widely accepted that in pathological conditions such as placental insufficiency, toxemia of pregnancy and maternal smoking, placental blood flow significantly decreases. The human placenta is a non-innervated organ and the local regulatory mechanism of the placental vasoactivity has not been fully understood as yet. But adenosine is reported to be a potent vasodilator also in human placental vessels (10), and our results confirmed that placental nucleotidases, especially $5^{\prime}-\mathrm{N}$, were confined to the microvillous membrane adjacent to the maternal circulation. Not only in such pathological conditions 


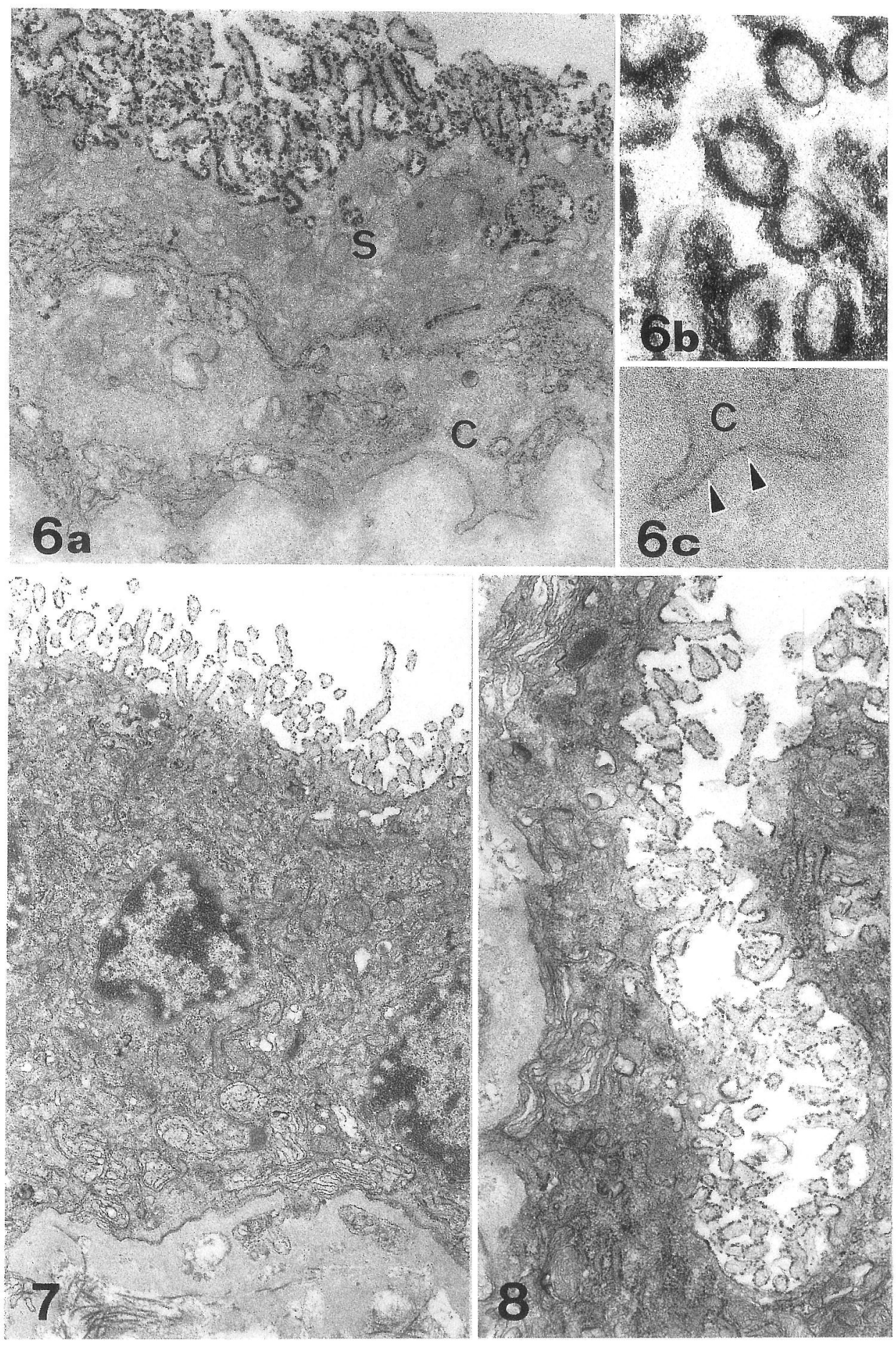


as mentioned above, but also in normal uterine contractions during labor pains, the placental blood flow significantly decreases and thus local ischemia in the trophoblast might occur. Regarding this phenomenon, it was reported that the adenosine concentration in the placental tissue increased a hundred times after the onset of labor compared to that in the prelabored placenta (22). This increased concentration of adenosine might act as a vasodilator on the placental vessels. In this context, it is possible to assume that human placental $5^{\prime}-\mathrm{N}$, similar to $5^{\prime}-\mathrm{N}$ in the ischemic myocardial tissue, receiving maternal information such as hypoxia, acidosis and some other factors, may serve as a regulator of the placental vasoactivity.

Third, the placental nucleotidase system, producing adenosine, may modulate the placental function by changing the intracellular cyclic AMP level. Cyclic nucleotide is shown to play an important role in the placental physiology (16) and the authors have already ultracytochemically demonstrated the presence of ACLase activity in the human placenta (15). At the same time, the presence of adenosine sensitive-site on ACLase in several tissues has already been proved (12), and adenosine receptor has been shown to also exist in the human placenta, which alters ACLase activity in this tissue (7). These facts lead us to assume that adenosine, generated by nucleotidases, especially by $5^{\prime}-\mathrm{N}$, may be of significance in the regulation of placental functions by affecting ACLase activity, as well as by directly acting as a platelet anti-aggregator or a vasodilator.

In conclusion, direct cytochemical evidence of the localizations of the placental nucleotidase activities has been demonstrated, with special emphasis on that of $5^{\prime}-\mathrm{N}$ activity. At the same time, it was found that AOPCP could be applied cytochemically as a potent inhibitor of $5^{\prime}-\mathrm{N}$ activity. The results of the present study suggest that the syncytiotrophoblast is active in the nucleotide metabolism, and that this nucleotidase system, especially $5^{\prime}-\mathrm{N}$, might play a role in the physiology of placental functions by maintaining the placental microcirculation or affecting the cyclic-AMP level in the syncytiotrophoblast, although the details are as yet unknown.

Figs. 6a-c. Ultracytochemical localization of $\mathrm{Ca}^{++}$ATPase in the human term placenta.

a. $\mathrm{Ca}^{++} \mathrm{ATPase}$ activity, unstained specimen. $\times 17,000$

The reaction products were observed strongly on the microvillous membrane and weakly on the basal plasma membrane of the syncytium (S). Very faint precipitates could be observed on the plasma membrane of the cytotrophoblast $(\mathrm{C})$. Activity could also be found on the plasma membrane of the pinocytotic vesicles of the syncytiotrophoblast.

b. Activity on the microvillous membrane of the syncytium, at higher magnification, stained specimen. $\times 48,000$

Strong activity was found.

c. Precipitates on the plasma membrane of the cytotrophoblast (C), at higher magnification, unstained specimen. $\quad \times 42,000$

Very faint precipitates were observed on the cytotrophoblastic plasma membrane (arrow heads).

FIG. 7. Ultracytochemical localization of $\mathrm{Mg}^{++}$ATPase. $\quad \times 9,500$

Activity on the microvillous membrane could be recognized. Fine precipitates could also be found on the basal plasma membrane, especially on the basal infoldings of the syncytium, but they are so faint that we cannot, at present, decide whether these precipitates indicate true enzymatic reaction or not.

FIG. 8. Ultracytochemical localization of NDPase, using ADP as a substrate. $\quad \times 20,000$ Activity was confined to the microvillous membrane of the syncytium. 


\section{ACKNOWLEDGMENTS}

The authors are very grateful to Tsunehiko Hanihara for his advice. The authors would like to thank Ms. A. Yamakawa, Ms. K. Onozaki and Ms. S. Komuro for their excellent technical assistance.

\section{REFERENCES}

1. Ando, T., Fujimoto, K., Mayahara, H. and Ogawa, K.: A new one step method for the histochemistry and cytochemistry of $\mathrm{Ca}^{++}$ATPase activity. Acta histochem. cytochem. 14; 705726, 1981 .

2. Barradas, M., Khokher, M., Hutton, R., Craft, I. L. and Dandona, P.: Adenosine diphosphate-degrading activity in placenta. Clin. Sci. 64; 239-241, 1983.

3. Berne, R. M.: The role of adenosine in the regulation of coronary blood flow. Circ. Res. 47; 807-813, 1980 .

4. Borgers, M.: The cytochemical application of new potent inhibitors of alkaline phosphatase. J. Histochem. Cytochem. 21; 812-824, 1973.

5. Born, G. V. R. and Cross, M. J.: The aggregation of blood platelets. J. Physiol. 168; 178195, 1963.

6. Burger, R. M. and Lowenstein, J. M.: Preparation and properties of 5 '-nucleotidase from smooth muscle of small intestine. J. Biol. Chem. 245; 6274-6280, 1970.

7. Fox, I. H. and Kurpis, L.: Binding characteristics of an adenosine receptor in human placenta. J. Biol. Chem. 258; 6952-6955, 1983.

8. Hagiwara, H., Ohtsu, Y., Shimonaka, M. and Inada, Y.: $\mathrm{Ca}^{2+}$-or $\mathrm{Mg}^{2+}$-dependent ATPase in plasma membrane of cultured endothelial cells from bovine carotid artery. Biochim. Biophys. Acta 734; 133-136, 1983.

9. Hutton, R. A., Chow, F. P. R., Craft, I. L. and Dandona, P.: Inhibitors of platelet aggregation in the feto-placental unit and myometrium with particular reference to the ADP-degrading property of placenta. Placenta 1; 125-130, 1980.

10. Krygics, J., Urban, J. and Malofiejew, M.: Influence of adenosine on contractility of isolated vessels of human placenta. Am. J. Obstet. Gynecol. 131; 587-589, 1978.

11. Kurahashi, K. and Maeda, T.: Fine structural localization of specific phosphatase in special sensory neuron of rat. Acta histochem. cytochem. 8; 72, 1975.

12. Londos, C. and Wolff, J.: Two distinct adenosine-sensitive sites on adenylate cyclase. Proc. Natl. Acad. Sci. 74; 5482-5486, 1977.

13. Maguire, M. H., Krishnakantha, T.P. and Aronson, D. M.: Human placental 5'nucleotidase: purification and properties. Placenta 5; 21-40, 1984.

14. Matsubara, S., Tamada, T. and Saito, T.: Ultracytochemical localization of alkaline phosphatase and acid phosphatase activities in the human term placenta. Acta histochem. cytochem. 20; 1987. (in press)

15. Matsubara, S., Tamada, T. and Satio, T.: Ultracytochemical localization of adenylate cyclase and guanylate cyclase activities in the human term placenta. Acta Obst. Gynaec. Jpn. 39; 10471053, 1987.

16. Milewich, L., Hendricks, T. S., Graham, J. E., Gant, N. F., Schwartz, B. E. and ManDonald, P. C.: Adenylate cyclase from term placenta and its regulation. Placenta 3; 165-180, 1982.

17. Novikoff, A. B. and Goldfischer, S.: Nucleotide diphosphatase activity in the Golgi apparatus and its usefulness for cytochemical studies. Proc. Natl. Acad. Sci. USA 47; 802-810, 1961.

18. Ogawa, K. S., Fujimoto, K. and Ogawa, K.: Ultracytochemical studies of adenosine nucleotidases in aortic endothelial and smooth muscle cells- $\mathrm{Ca}^{2+}$-ATPase and $\mathrm{Na}^{+}, \mathrm{K}^{+}-\mathrm{AT}$ Pase-. Acta histochem. cytochem. 19; 601-620, 1986. 
19. Pearson, J. D., Carleton, J. S. and Gordon, J. L.: Metabolism of adenosine nucleotides by ectoenzymes of vascular endothelial and smooth muscle cells in culture. Biochem. J. 190; 421-429, 1980.

20. Robinson, J. M. and Karnovsky, M. J.: Ultrastructural localization of 5 '-nucleotidase in guinea pig neutrophils based upon the use of cerium as capturing agent. J. Histochem. Cytochem. 31; 1190-1196, 1983.

21. Shami, Y. and Radde, I. C.: The effect of the $\mathrm{Ca}^{2+} / \mathrm{Mg}^{2+}$ concentration ratio on placental $\left(\mathrm{Ca}^{2+}-\mathrm{Mg}^{2+}\right)$-ATPase activity. Biochim. Biophys. Acta 255; 675-679, 1972.

22. Sim, M. K. and Maguire, M. H.: Presence of adenosine in the human term placenta, determination of adenosine content and pathways of adenosine metabolism. Circ. Res. 31; 779-788, 1972.

23. Wachstein, M. and Meisel, E.: Histochemistry of hepatic phosphatases at a physiologic $\mathrm{pH}$. With special reference to the demonstration of bile canaliculi. Am. J. Clin. Pathol. 27; 13-23, 1957.

24. Whitsett, J. A.: Specialization of plasma membrane of the human placenta. J. Pediatr. 96; 600-604, 1980. 